Scientific Paper

\title{
Comparison of treatment plans: a retrospective study by the method of radiobiological evaluation
}

\author{
Niyas PUZHAKKAL ${ }^{1,2,3, a}$, Abdullah KALLIKUZHIYIL KoCHUNNY ${ }^{1}$, Noufal MANTHALA PAdANNAYIL ${ }^{1,2,3}$, Navin \\ SINGH ${ }^{4}$, Jumanath ELAVAN CHALIL ${ }^{3}$, Jamshad KULANGARAKATH UMER ${ }^{2}$ \\ ${ }^{1}$ Department of Physics, Farook College, Kozhikode-673632, Kerala, India \\ ${ }^{2}$ Department of Medical Physics and Radiation Oncology, Baby Memorial Hospital, Kozhikode-673004, Kerala, India \\ ${ }^{3}$ Department of Physics, University of Calicut, Malappuram-673635, Kerala, India \\ ${ }^{4}$ Department of Radiotherapy, King George's Medical University, Lucknow, UP, India \\ ${ }^{a}$ E-mail address: pniyas@gmail.com
}

(received 17 April 2016; revised 29 July 2016; accepted 29 August 2016)

\begin{abstract}
There are many situations in radiotherapy where multiple treatment plans need to be compared for selection of an optimal plan. In this study we performed the radiobiological method of plan evaluation to verify the treatment plan comparison procedure of our clinical practice. We estimated and correlated various radiobiological dose indices with physical dose metrics for a total of 30 patients representing typical cases of head and neck, prostate and brain tumors. Three sets of plans along with a clinically approved plan (final plan) treated by either Intensity Modulated Radiation Therapy (IMRT) or Rapid Arc (RA) techniques were considered. The study yielded improved target coverage for final plans, however, no appreciable differences in doses and the complication probabilities of organs at risk were noticed. Even though all four plans showed adequate dose distributions, from dosimetric point of view, the final plan had more acceptable dose distribution. The estimated biological outcome and dose volume histogram data showed least differences between plans for IMRT when compared to RA. Our retrospective study based on 120 plans, validated the radiobiological method of plan evaluation. The tumor cure or normal tissue complication probabilities were found to be correlated with the corresponding physical dose indices.
\end{abstract}

Key words: plan evaluation; EUD; TCP; NTCP; DVH.

\section{Introduction}

The modern technological developments have introduced remarkable improvements in planning and execution of radiotherapy. The Intensity Modulated Radiation Therapy (IMRT) ensures a highly conformal dose distribution to the target and is very beneficial when the target and the critical structures are situated near or overlapping each other [1]. The conformal treatment plans are produced by an inverse planning optimization algorithm associated with a Treatment Planning System (TPS). Depending on the number of beams, their directions and given dose constraints, the iterative optimization algorithm generates a solution in the form of treatment plans. Thus, the optimization engine of IMRT planning allows the planner to produce multiple number of treatment plans. Out of these, the best plan is selected by performing a detailed plan comparison.

In the routine clinical practice, different tools are available for the selection of the most suitable treatment plans. A commonly used method for evaluation of treatment plan is by judging physical quantities such as dose and dose-volume parameters. This plan assessment process includes 1) viewing the two-dimensional dose distributions calculated on computed tomography images and 2) examining Dose-Volume Histograms (DVHs) for the maximum, mean and different clinically relevant volume doses for each Organs At Risk (OARs) or tumor. In addition to this, there is another method of evaluation which is based on radiobiological dose-response models. In this assessment, acceptance or rejection of a plan is done by use of radiobiological indices such as Tumor Control Probability (TCP) and Normal Tissue Complication Probability (NTCP). It has been studied that the treatment plans with identical mean, maximum or minimum doses may have significantly different clinical outcomes [2]. This necessitates the use of an additional plan evaluation tool such as radiobiological estimates along with the conventional dosimetric-based evaluation.

In contrast to the three dimensional conformal radiotherapy plans, the dose distribution of IMRT plans is more heterogeneous and complex in nature [3-5]. In order to select an optimum plan, the clinician needs an effective plan comparison method to grade the plans in terms of relevant dosimetric quantities. A detailed investigation of the dose 
distributions, DVHs and radiobiological estimation will be required for finding the most suitable treatment plan. However, in the current clinical practice, the execution of whole plan assessment methods is a time-consuming task and thereby, a visual inspection of the dose distribution and DVHs are carried out. In the present study, we performed the radiobiological method of evaluations along with the routine physical dose evaluation for a number of patients who were treated in our radiation therapy center. The main purpose of this study was to re-check the comparison of multiple treatment plans obtained for a particular patient by use of radiobiological response evaluation. We estimated the TCP and NTCP in both IMRT and Rapid Arc (RA) plans and we correlated these radiobiological estimations with the physical dose metrics.

\section{Material and Methods}

\section{Patient plans}

A total of 30 patients representing typical cases of Head and Neck $(H \& N)$, prostate and brain tumors at our institution were used in this retrospective planning study. All the plans were designed on Eclipse TPS (Varian Medical Systems, Palo Alto, CA, USA), version 10.0, using Anisotropic Analytical dose calculation Algorithm. These treatment plans were selected at random from the cohort of patients with different carcinomas, who had already completed their treatments with use of a Varian Clinac-iX Linear Accelerator (LINAC) with a 120 leaf millennium multi leaf collimator (Varian Medical Systems, Palo Alto, CA). Three sets of plans (namely: plan 1, plan 2 and plan 3) along with a clinically approved and verified plan (final plan) were studied for each patient. These competing plans were generated by using slightly different objective functions, which may be considered acceptable. All four plans of one patient were prepared in the same type of technique (IMRT/RA). Our 10 cases of each group consisted of 5 patients with IMRT technique and 5 patients with RA technique. Thus, a total of 120 treatment plans from 30 patients were selected for analysis, which contained both IMRT and RA plans in equal number. The IMRT plans generally consisted of either seven or nine static beams, where as the most of RA plans were created by using two arcs (either full or partial), rotating in opposite directions. All of the investigated prostate cases were treated with a dose of $250 \mathrm{cGy} /$ fraction, giving a total dose of 7000 cGy. The H\&N patients were treated with $212.1 \mathrm{cGy} /$ fraction (total dose $=7000 \mathrm{cGy}$ ). The prescription dose for brain cases was 5000 cGy delivered in 25 fractions (phase 1) followed by $1000 \mathrm{cGy}$ in another 5 fractions (phase 2). Figure 1 depicts typical dose distributions of the $\mathrm{H} \& \mathrm{~N}$, prostate and brain treatment plans.

\section{Plan analysis}

Plans in each group were compared against their final plan by DVH analysis. The Planning Target Volume (PTV) of each site included its primary tumor. Other high and low risk lymph nodes were also delineated, but were not included in the present dosimetric study. For target coverage, at least $95 \%$ of the PTV should receive at least $95 \%$ of the prescribed dose. We analyzed the dose to $95 \%$ of the volume $\left(\mathrm{D}_{95 \%}\right)$ for all PTVs and the maximum dose $\left(D_{\max }\right)$ or mean dose $\left(D_{\text {mean }}\right) /$ dose to volumes of different OARs. During the treatment planning process, various OAR dose constraints were used as per Radiation Therapy Oncology Group (RTOG) and Quantitative Analysis of Normal Tissue Effects in the Clinic (QUANTEC) protocols. These constraints included no more than $50 \%$ of bladder volume to receive a dose greater than $6500 \mathrm{cGy}\left(\mathrm{V}_{50} \leq\right.$ $65 \%$ ), no more than $50 \%$ of rectum to receive a dose of 5000 cGy $\left(\mathrm{V}_{50}<50 \%\right)$ and different $\mathrm{D}_{\max } / \mathrm{D}_{\text {mean }}$ for rest of the OARs. All these figures are summarized in Table 1 [6-15].

Table 1. Normal tissue tolerance for conventional fractionation.

\begin{tabular}{lccc}
\hline \hline Organ & \multicolumn{2}{c}{ Dose values [cGy] } & References \\
\hline Brainstem & $D_{\max }$ & 5400 & {$[6]$} \\
Optic chiasm & $D_{\max }$ & 5500 & {$[7]$} \\
and Optic nerve & $D_{\max }$ & 5000 & {$[8]$} \\
Spinal cord & $D_{\operatorname{mean}}$ & 4500 & {$[9]$} \\
Cochlea & $\mathrm{D}_{\max }$ & 7000 & {$[10]$} \\
Mandible & $\mathrm{D}_{\max }$ & 1200 & {$[11]$} \\
Lens & $\mathrm{D}_{\operatorname{mean}}$ & 2500 & {$[12]$} \\
Parotid & $\mathrm{D}_{\max }$ & 5200 & {$[13]$} \\
Small bowel & $\mathrm{V}_{50}$ & 6500 & {$[14]$} \\
Bladder & $\mathrm{V}_{50}$ & 5000 & {$[15]$} \\
Rectum & $\mathrm{D}_{\max }$ & 5200 & {$[10]$} \\
Femoral head & &
\end{tabular}
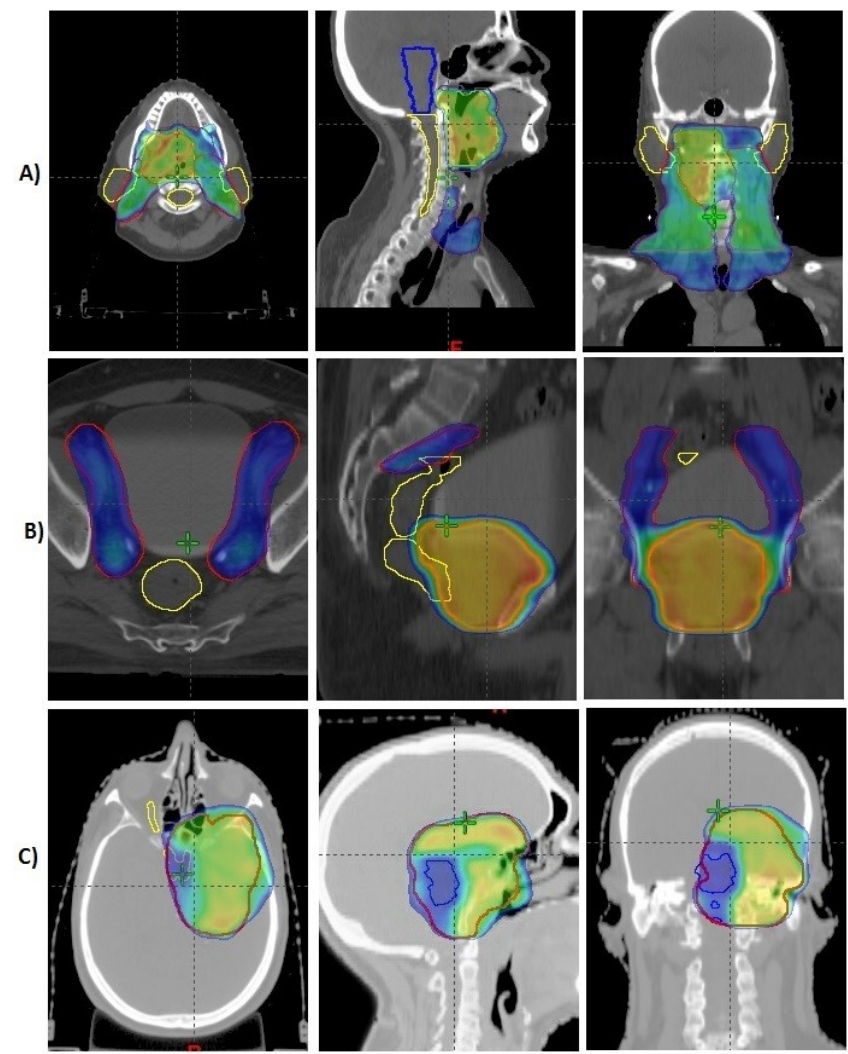

Figure 1. Axial, sagittal and coronal views (from left to right) of color washed isodose distributions of a A) H\&N patient, B) prostate patient and C) brain patient. 
For the comparison of treatment plans, a radiobiological based plan evaluation was also carried out. There are different models available in literature for the prediction of tumor cure and normal tissue complication probabilities [16-20]. In the present study, we used Equivalent Uniform Dose (EUD) based radiobiological modelling, which is very effective in predicting the effect of more heterogeneous dose distributions [21]. The EUD is the uniform dose that gives the same radiobiological effect, if delivered over the same number of fractions as does the non-uniform dose distribution of interest. According to Niemierko's model, the EUD is given by [21-22]:

$$
E U D=\left(\sum_{i=1}\left(v_{i} D_{i}^{a}\right)\right)^{\frac{1}{a}}
$$

where $\mathrm{a}$ is unitless parameter, which describes the volumetric dependence of dose-response curve. $v_{i}$ is also unitless and represents the $i^{\text {th }}$ partial volume receiving a dose $D_{i}$ in Gy. The TCP and NTCP are calculated by the following equations [21]:

$$
\begin{gathered}
T C P=\frac{1}{1+\left(\frac{T C D_{50}}{E U D}\right)^{4 \gamma_{50}}} \\
N T C P=\frac{1}{1+\left(\frac{T D_{50}}{E U D}\right)^{4 \gamma_{50}}}
\end{gathered}
$$

$\mathrm{TCD}_{50}$ is the dose required to control $50 \%$ of the tumor and $\mathrm{TD}_{50}$ is the tolerance dose of normal tissues for a $50 \%$ complication rate when the tumor and normal tissues are homogeneously irradiated. $\gamma_{50}$ is a dimensionless model parameter, which represents the slope of the dose-response curve. The EUD-based calculations were performed by using a freely available MatLab program (The MathWorks, Inc., Natick, MA, USA), Eudmodel.m [21]. The cumulative DVHs of 120 plans exported from Eclipse along with various radiobiological factors (Table 2) for different sites were given as input to the above program [23-27].

\section{Statistical analysis}

For examining the significance of the results obtained during plan comparison, statistical tests have been carried out. The variations in both target coverage and OAR doses across four plans were statistically studied by using one way ANOVA. The differences between data were considered statistically significant, if denoted by small $\mathrm{p}$ values $(<0.05)$. Also, the correlation between physical and radiobiological dose indices was calculated by parametric Pearson tests and the correlation coefficients were considered statistically significant for $\mathrm{p}$-value less than 0.05 .

\section{Results}

The treatment plan DVHs are compared for all of the investigated plans. The detailed analysis of DVH data results in the following dosimetric information. Figure 2 compares the target coverage of different plans in terms of both the $\mathrm{D}_{95 \%}$ and EUD of the PTVs for all treatment sites. It is observed that, the values of $\mathrm{D}_{95 \%}$ and EUD increased in the final plan while comparing with that of other plans. The observed maximum differences in the average values of $\mathrm{D}_{95 \%}$ between final plan and a plan in the same group were $0.85 \%, 2.11 \%$ and $3.14 \%$ for the $H \& N$, prostate and brain plans, respectively. The corresponding EUD values of PTV were 1.99\%, $1.97 \%$ and $6.04 \%$ for the above study groups.

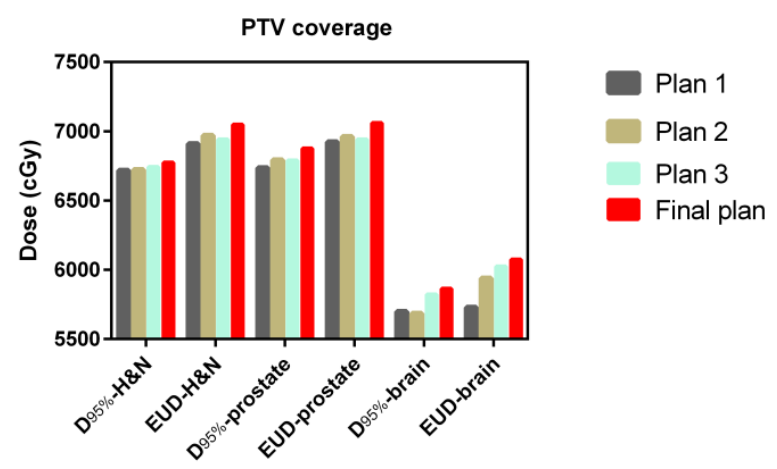

Figure 2. PTV coverage in terms of D95\% and EUD for different

\begin{tabular}{|c|c|c|c|c|c|c|c|}
\hline Structure set & Volume Type & End point & $\mathbf{a}$ & $\mathbf{T C D}_{50} / \mathbf{T D}_{50}$ & $\gamma_{50}$ & $\alpha / \boldsymbol{B}$ & References \\
\hline H\&N-PTV & Tumor & - & -13 & 51.77 & 2.28 & 10 & {$[23]$} \\
\hline Prostate-PTV & Tumor & - & -10 & 28.34 & 1 & 1.2 & [24] \\
\hline Brain-PTV & Tumor & - & -8 & 27.04 & 0.75 & 10 & {$[25]$} \\
\hline Brainstem & Normal & Necrosis & 7 & 65 & 3 & 2.1 & {$[21,26]$} \\
\hline Spinal cord & Normal & Myelitis & 13 & 66.5 & 4 & 2 & {$[21,27]$} \\
\hline Parotid & Normal & Xerostomia & 0.5 & 46 & 4 & 2 & [23] \\
\hline Mandible & Normal & Reduced joint junction & 10 & 72 & 4 & 3.5 & [23] \\
\hline Bladder & Normal & Volume loss & 2 & 80 & 4 & 8 & [24] \\
\hline Rectum & Normal & Necrosis & 8.33 & 80 & 4 & 3.9 & {$[24]$} \\
\hline Femoral head & Normal & Necrosis & 4 & 65 & 4 & 0.85 & {$[24]$} \\
\hline Small bowel & Normal & Obstruction & 6 & 55 & 4 & 3 & {$[23]$} \\
\hline Optic chiasm & Normal & Blindness & 25 & 65 & 3 & 3 & {$[21,26]$} \\
\hline Optic nerve & Normal & Blindness & 25 & 65 & 3 & 3 & {$[21,26]$} \\
\hline Lens & Normal & Cataracts & 3 & 18 & 1 & 1.2 & {$[21]$} \\
\hline Cochlea & Normal & Chronic serous otitis & 31 & 65 & 3 & 3 & {$[23]$} \\
\hline
\end{tabular}
plans in each study group.

Table 2. List of parameters used for calculation of EUD-based TCP and NTCP. 
Table 3. Detailed report on comparison between different dose values and EUD for various OARs across all 4 plans in each group. Statistical analysis, one way ANOVA was carried out. No statistically significant variations in dose figures were observed ( $p>0.05$ ).

\begin{tabular}{|c|c|c|c|c|c|c|c|}
\hline \multirow{2}{*}{$\begin{array}{l}\text { Study } \\
\text { groups }\end{array}$} & \multirow{2}{*}{ Structure set } & \multirow{2}{*}{$\begin{array}{c}\text { Dose } \\
\text { indices }\end{array}$} & \multicolumn{4}{|c|}{ Average \pm standard deviation dose values in cGy } & \multirow{2}{*}{$p$ value } \\
\hline & & & Plan 1 & Plan 2 & Plan 3 & Final plan & \\
\hline \multirow{10}{*}{$\mathrm{H} \& \mathrm{~N}$} & \multirow{2}{*}{ Brainstem } & $\mathrm{D}_{\max }$ & $4975.8 \pm 289.8$ & $4929.1 \pm 381.7$ & $4946.6 \pm 359.9$ & $5010.0 \pm 228.9$ & 0.6739 \\
\hline & & EUD & $2733.4 \pm 406.9$ & $2705.4 \pm 409.4$ & $2742.0 \pm 348.1$ & $2743.9 \pm 371.4$ & 0.6086 \\
\hline & \multirow{2}{*}{ Spinal cord } & $\mathrm{D}_{\max }$ & $4233.3 \pm 305.1$ & $4283.0 \pm 327.2$ & $4200.5 \pm 357.0$ & $4239.8 \pm 297.8$ & 0.3053 \\
\hline & & EUD & $2735.5 \pm 280.5$ & $2689.5 \pm 270.6$ & $2659.5 \pm 308.4$ & $2733.6 \pm 306.6$ & 0.1829 \\
\hline & \multirow{2}{*}{ Right parotid } & $\mathrm{D}_{\text {mean }}$ & $3632.3 \pm 836.2$ & $3667.6 \pm 315.0$ & $3688.1 \pm 816.4$ & $3537.0 \pm 845.9$ & 0.1859 \\
\hline & & EUD & $2714.3 \pm 946.1$ & $2733.2 \pm 926.3$ & $2754.5 \pm 936.2$ & $2754.5 \pm 936.2$ & 0.7449 \\
\hline & \multirow{2}{*}{ Left parotid } & $\mathrm{D}_{\text {mean }}$ & $3620.5 \pm 651.8$ & $3608.6 \pm 660.5$ & $3579.5 \pm 684.3$ & $3564.9 \pm 689.7$ & 0.4689 \\
\hline & & EUD & $2676.0 \pm 677.0$ & $2659.0 \pm 690.6$ & $2657.8 \pm 721.7$ & $2621.7 \pm 720.2$ & 0.5694 \\
\hline & \multirow{2}{*}{ Mandible } & $\mathrm{D}_{\max }$ & $7426.3 \pm 348.2$ & $7498.5 \pm 390.7$ & $7502.7 \pm 376.3$ & $7372.4 \pm 307.0$ & 0.1724 \\
\hline & & EUD & $5675.2 \pm 551.8$ & $5720.0 \pm 537.3$ & $5699.5 \pm 556.8$ & $5632.7 \pm 518.1$ & 0.2658 \\
\hline \multirow{10}{*}{ Prostate } & \multirow{2}{*}{ Bladder } & $\mathrm{V}_{50}$ & $4110.2 \pm 451.1$ & $4104.7 \pm 441.3$ & $4083.2 \pm 477.6$ & $4161.8 \pm 401.2$ & 0.8434 \\
\hline & & EUD & $3976.7 \pm 433.8$ & $4014.9 \pm 426.6$ & $3992.2 \pm 431.4$ & $4024.2 \pm 416.0$ & 0.6061 \\
\hline & \multirow{2}{*}{ Rectum } & $\mathrm{V}_{50}$ & $4284.7 \pm 669.5$ & $4198.0 \pm 583.0$ & $4165.2 \pm 595.7$ & $4258.5 \pm 518.2$ & 0.5301 \\
\hline & & EUD & $5311.7 \pm 367.2$ & $5367.1 \pm 386.7$ & $5439.2 \pm 322.2$ & $5401.5 \pm 276.7$ & 0.1171 \\
\hline & \multirow{2}{*}{$\begin{array}{l}\text { Right femoral } \\
\text { head }\end{array}$} & $\mathrm{D}_{\max }$ & $4607.8 \pm 459.3$ & $4703.7 \pm 365.2$ & $4658.1 \pm 433.2$ & $4708.8 \pm 383.9$ & 0.6116 \\
\hline & & EUD & $1793.2 \pm 404.5$ & $1810.8 \pm 372.0$ & $1832.0 \pm 339.0$ & $1817.5 \pm 312.3$ & 0.6234 \\
\hline & \multirow{2}{*}{ Left femoral head } & $\mathrm{D}_{\max }$ & $4703.9 \pm 417.8$ & $4689.5 \pm 434.9$ & $4640.7 \pm 577.1$ & $4722.8 \pm 439.5$ & 0.7252 \\
\hline & & EUD & $1763.9 \pm 475.1$ & $1817.3 \pm 377.3$ & $1798.1 \pm 344.8$ & $1787.0 \pm 324.6$ & 0.5428 \\
\hline & \multirow{2}{*}{ Small bowel } & $\mathrm{D}_{\max }$ & $5495.3 \pm 818.9$ & $5632.5 \pm 572.8$ & $5525.0 \pm 652.1$ & $5634.6 \pm 576.7$ & 0.5934 \\
\hline & & EUD & $3085.6 \pm 333.8$ & $3141.4 \pm 227.1$ & $3127.1 \pm 268.5$ & $3147.9 \pm 238.1$ & 0.3561 \\
\hline \multirow{16}{*}{ Brain } & \multirow{2}{*}{ Brainstem } & $\mathrm{D}_{\max }$ & $5358.1 \pm 520.2$ & $5368.6 \pm 537.0$ & $5410.6 \pm 531.9$ & $5353.2 \pm 544.9$ & 0.4714 \\
\hline & & EUD & $3852.4 \pm 1215.1$ & $3838.8 \pm 1198.2$ & $3813.8 \pm 1002.4$ & $3708.6 \pm 1081.8$ & 0.5353 \\
\hline & \multirow{2}{*}{ Optic chiasm } & $\mathrm{D}_{\max }$ & $5153.3 \pm 522.9$ & $5096.8 \pm 494.9$ & $5074.5 \pm 452.0$ & $5105.5 \pm 432.8$ & 0.5093 \\
\hline & & EUD & $4335.3 \pm 996.0$ & $4384.1 \pm 754.4$ & $4353.0 \pm 727.6$ & $4392.3 \pm 710.8$ & 0.8439 \\
\hline & \multirow{2}{*}{ Right optic nerve } & $\mathrm{D}_{\max }$ & $3911.2 \pm 1770.9$ & $3838.9 \pm 1726.2$ & $3732.8 \pm 1739.4$ & $3913.0 \pm 1695.9$ & 0.2527 \\
\hline & & EUD & $3044.4 \pm 1737.8$ & $2989.4 \pm 1674.7$ & $2857.3 \pm 1490.3$ & $2897.8 \pm 1461.4$ & 0.2844 \\
\hline & \multirow{2}{*}{ Left optic nerve } & $\mathrm{D}_{\max }$ & $4532.1 \pm 1888.2$ & $4546.9 \pm 1902.8$ & $4137.5 \pm 2102$ & $4485.6 \pm 1916.3$ & 0.3660 \\
\hline & & EUD & $3822.9 \pm 1864.3$ & $3847.6 \pm 1893.2$ & $3637.0 \pm 1786.2$ & $3624.3 \pm 1779.6$ & 0.1316 \\
\hline & \multirow{2}{*}{ Right lens } & $\mathrm{D}_{\max }$ & $765.1 \pm 301.5$ & $797.8 \pm 351.8$ & $840.7 \pm 359.8$ & $796.5 \pm 343.8$ & 0.3022 \\
\hline & & EUD & $295.0 \pm 124.2$ & $310.7 \pm 160.6$ & $329.6 \pm 166.7$ & $301.9 \pm 148.1$ & 0.2435 \\
\hline & \multirow{2}{*}{ Left lens } & $\mathrm{D}_{\max }$ & $1004.5 \pm 551.0$ & $1009.6 \pm 547.9$ & $1018.1 \pm 531.9$ & $1001.6 \pm 538.0$ & 0.8828 \\
\hline & & EUD & $415.7 \pm 285.4$ & $431.8 \pm 300.6$ & $431.4 \pm 337.7$ & $428.2 \pm 333.9$ & 0.7383 \\
\hline & \multirow{2}{*}{ Right cochlea } & $\mathrm{D}_{\text {mean }}$ & $3659.7 \pm 2424.8$ & $3632.1 \pm 2448.2$ & $3602.2 \pm 2400.5$ & $3635.0 \pm 2433$ & 0.6530 \\
\hline & & EUD & $2982.5 \pm 2292.5$ & $2913.5 \pm 2295.3$ & $2789.2 \pm 2100.2$ & $2785.2 \pm 2138.3$ & 0.2720 \\
\hline & \multirow{2}{*}{ Left cochlea } & $\mathrm{D}_{\text {mean }}$ & $4309.1 \pm 2657.4$ & $4244.6 \pm 2724.6$ & $4207.5 \pm 2737.8$ & $4222.5 \pm 2732.8$ & 0.1923 \\
\hline & & EUD & $3916.2 \pm 2694.3$ & $3736.6 \pm 2625.8$ & $3741.2 \pm 2642.0$ & $3708.8 \pm 2641.7$ & 0.1970 \\
\hline
\end{tabular}

The comparison results of physical and biological dose indices of various OARs were shown in Table 3 in terms of average \pm standard deviation dose values. The study is also extended between IMRT and RA plans of each group of patient. The maximum percentage differences of $\mathrm{D}_{95 \%}$ of PTV in RA plans were $2.1 \%, 2.5 \%$ and $2.3 \%$ for $\mathrm{H} \& \mathrm{~N}$, prostate and brain cases, respectively. However, the corresponding deviations of IMRT plans were $0.86 \%, 0.85 \%$ and $0.91 \%$ only. The radiobiological estimation of EUD and TCP showed a similar behaviour. The estimated maximum percentage variations in EUD (TCP) of RA plans were $2.3 \%(1.4 \%), 2.6 \%(0.25 \%)$ and $12.4 \%(4.8 \%)$ and those of IMRT plans were $1.9 \%(0.9 \%), 1.3 \%(0.14 \%)$ and $0.52 \%(0.13 \%)$, respectively, for the above study groups.

\section{Discussion}

The overall objective of this retrospective study is to check the acceptability of treatment plans by incorporating the radiobiological evaluation tools. The results of the dosimetric comparison of 120 radiotherapy plans, across various sites and treated by two prominent methods, support the plan assessment process in the routine practice. Even though all four plans showed passable dose distributions, from dosimetric point of view, the final plan had more acceptable dose distribution when compared to other plans. This study revealed that the final plans showed higher target coverage, which is represented in terms of $\mathrm{D}_{95 \%}$ and EUD of PTV. The main aspect of this study is the use of radiobiological model for the treatment plan comparison. Radiobiological methods were reported to be effective in the plan evaluation process in comparison to the use of physical dose metrics alone [20]. The estimated TCP, which is in correlation with the target conformity index [20], did not show any considerable variation along different plans, as is evident in Figure 3.

Although the final plan in each group exhibited a slight improvement in PTV coverage, no statistically significant differences were observed. Based on the doses of various 
OARs shown in Table 3 , neither of the plans appears significantly different from the final plan. The calculated NTCPs for most of the structures were found to have little or no variation between the plans, which is clearly shown in Figure 4. Though relatively larger differences were observed for certain structures such as mandible, rectum, optic nerves and cochlea, none of these variations were statistically significant. The present study also reported the comparison of treatment plans for both IMRT and RA techniques. Figure 5 illustrates the maximum \% variation of both EUD and physical dose indices of final plan from other plans in IMRT and RA techniques. A minute deviation of the dosimetric parameters from final plan to any other plan was noticed for IMRT, whereas the final plans in RA technique showed relatively larger deviation from rest of the plans.

In the current retrospective planning study, we have performed a direct comparison between radiobiological and physical dose indices. The dose coverage of PTVs, in terms of $\mathrm{D}_{95 \%}$, of all plans was found to be correlated with the estimated TCPs of the corresponding plans, represented by the Pearson correlation coefficients (r). The diagonal elements of Table 4 represent a higher correlation between physical and biological dose metrics of every corresponding plans of each of the patient group. However, the off-diagonal elements denote the correlation of physical dose index of any plan with respect to the biological dose index of every other plan. A relatively lower correlation was noticed in most of these figures, which also confirmed the use of biological index, TCP, for dose coverage of PTVs in plan comparison process. Table 5 conveys the correlation between physical doses and NTCP of various OARs for final plans. The complication probabilities for different organs such as spinal cord, parotids, mandible, bladder, rectum, small bowel, optic chiasm, right optic nerve, lenses and left cochlea were correlated well with their physical dose indices $(\mathrm{p}<0.05)$. However, the correlations of $\mathrm{D}_{\max }$ received by brainstem in $\mathrm{H} \& \mathrm{~N}$ plans and that of femoral head, left optic nerve and the $\mathrm{D}_{\text {mean }}$ of right cochlea in other plans were deviated more with the radiobiological complication probabilities. The TCP or NTCP values depend on the radiobiological parameters and the dose distribution of the target or OARs. In this study, the same radiobiological parameters were used for all the plans of a particular group and the varying factor is the dose distribution of individual plans. The normal tissue structures located near or overlapping to the PTV were probably subjected to a significantly increased $D_{\max }$. In some of our investigated plans, the stated structures were found to be adjacent with the PTV and therefore their dose values deviated more with the radiobiological complication probabilities.

Conventional plan evaluation is based on single or multiple dose-volume constraints and one of its limitations is the requirement of more than one dose-volume point for evaluating the complication of a particular organ.

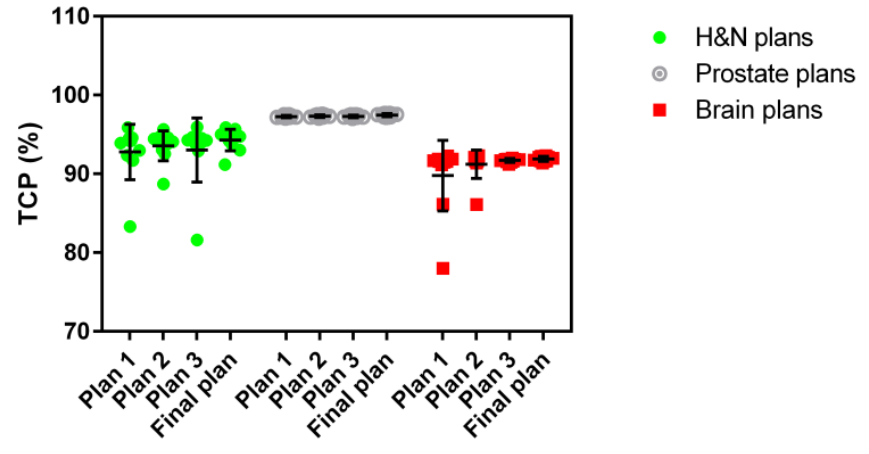

Figure 3. No major variations in TCP along different plans were observed.
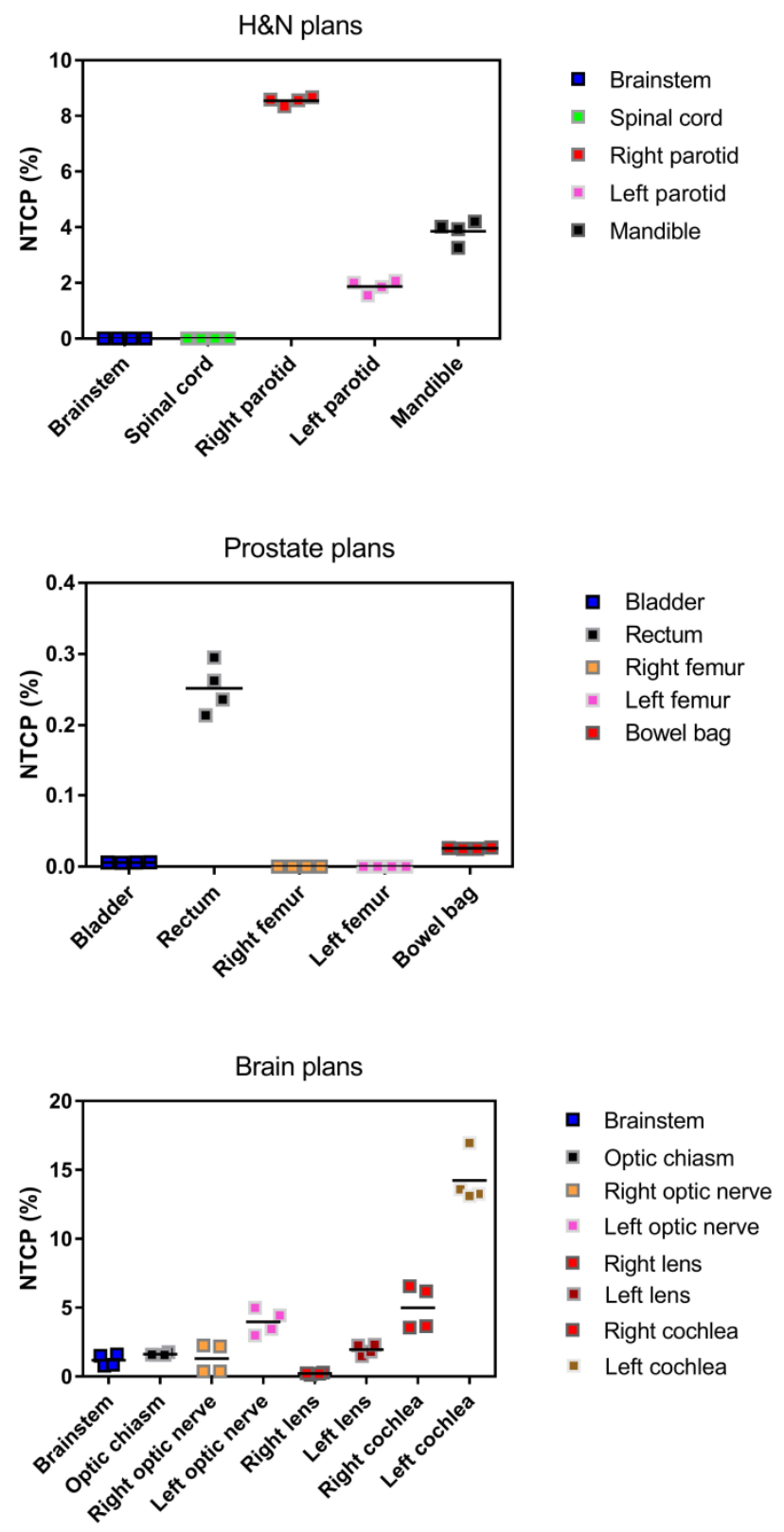

Figure 4. Estimated NTCP (\%) values of the various OARs were studied and average values for each set of plans were plotted. 

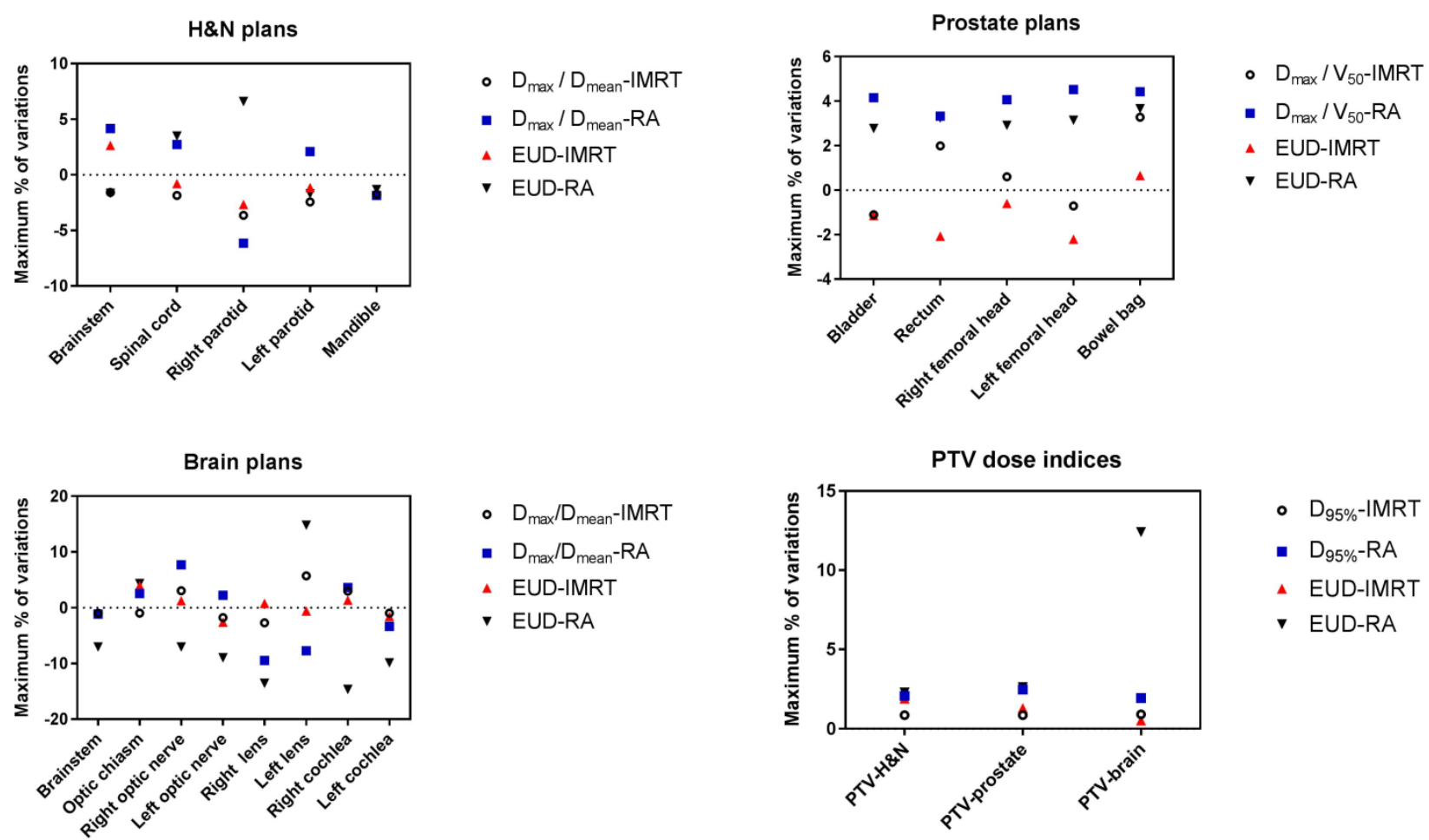

Figure 5. Deviation of final plan from other plans of IMRT and RA patients was studied in terms of both biological and physical dose indices. More discrepancies of target and OAR doses for RA plans than for IMRT were observed.

Table 4. Pearson correlation coefficient ( $r$ ) between TCP and $\mathbf{D}_{\mathbf{9 5} \%}$ of different PTVs.

\begin{tabular}{|c|c|c|c|c|c|}
\hline \multirow{2}{*}{ Study groups } & \multirow{2}{*}{$D_{95 \%}$} & \multicolumn{4}{|c|}{ TCP } \\
\hline & & Plan 1 & Plan 2 & Plan 3 & Final \\
\hline \multirow{4}{*}{ H\&N-PTV } & Plan 1 & 0.891 & -0.007 & -0.106 & -0.185 \\
\hline & Plan 2 & 0.258 & 0.869 & 0.063 & -0.010 \\
\hline & Plan 3 & 0.367 & 0.270 & 0.935 & 0.161 \\
\hline & Final & 0.103 & 0.200 & 0.212 & 0.921 \\
\hline \multirow{4}{*}{ Prostate-PTV } & Plan 1 & 0.575 & 0.444 & -0.031 & -0.124 \\
\hline & Plan 2 & 0.217 & 0.797 & 0.242 & 0.112 \\
\hline & Plan 3 & 0.582 & 0.757 & 0.538 & 0.331 \\
\hline & Final & 0.575 & 0.766 & 0.460 & 0.866 \\
\hline \multirow{4}{*}{ Brain-PTV } & Plan 1 & 0.895 & 0.769 & 0.089 & -0.014 \\
\hline & Plan 2 & 0.842 & 0.829 & 0.234 & 0.074 \\
\hline & Plan 3 & 0.116 & -0.195 & 0.725 & 0.668 \\
\hline & Final & 0.101 & -0.084 & 0.889 & 0.807 \\
\hline
\end{tabular}

During the plan evaluation the clinician needs to consider the priorities of various dose-volume constraints, when some of the constraints pass and other fails. However, radiobiological plan evaluation uses full three dimensional dose distributions, weighs different dose-volume criteria and results into a comprehensible estimate of biological outcome. Also, the plan assessment by using dose-volume criteria tells that an effect occur or do not occur with respect to certain dose limit. But the biological evaluation gives continuous estimates of tumor cure and organ complication probabilities rather than considering threshold levels in DVHs.
Table 5. The correlation between complication probabilities and physical dose values of various OARs were tabulated. Majority of the figures are statistically significant.

\begin{tabular}{llcc}
\hline \hline Study groups & Structure set & Pearson coefficient (r) & p-value \\
\hline \multirow{4}{*}{ H\&N } & Brainstem & 0.542 & 0.1059 \\
& Spinal cord & 0.875 & 0.0021 \\
& Right parotid & 0.889 & 0.0006 \\
& Left parotid & 0.731 & 0.0163 \\
& Mandible & 0.656 & 0.0393 \\
\hline \multirow{4}{*}{ Prostate } & Bladder & 0.846 & 0.0039 \\
& Rectum & 0.794 & 0.0061 \\
& Right femoral head & -0.440 & 0.1106 \\
& Left femoral head & -0.561 & 0.0784 \\
& Small bowel & 0.938 & 0.0001 \\
\hline \multirow{5}{*}{ Brain } & Brainstem & 0.824 & 0.0034 \\
& Optic chiasm & 0.708 & 0.0328 \\
& Right optic nerve & 0.643 & 0.0449 \\
& Left optic nerve & 0.447 & 0.1954 \\
& Right lens & 0.879 & 0.0307 \\
& Left lens & 0.834 & 0.0027 \\
& Right cochlea & 0.496 & 0.1446 \\
& Left cochlea & 0.692 & 0.0267 \\
\hline \hline
\end{tabular}

Hence, a properly calibrated radiobiological model is recommended as a tool to complement the conventional dosimetric analysis by predicting the radiobiological outcome, and particularly is useful when a clinician needs to select the best plan from competing plans. 
The comparison and ranking of treatment plans is of great significance as there are many cases in radiotherapy where multiple treatment plans need to be compared. There are few studies which have reported some ranking methods for plan evaluation and comparison. A study conducted by Miften et al. [5] developed an IMRT plan evaluation and ranking tool based on uncomplicated target conformity index (TCI+). The dosevolume-based indices sum up complex dose distributions to a single index, which can be used to choose the optimal plan. Another study performed by Akpati et al. [28] formulated a Unified Dosimetry Index (UDI) that reckons the deviations between treatment plans. This method uses an equation which is developed by combining different dosimetric objectives such as dose coverage, conformity, homogeneity and dose gradient. However, in this particular study, we have performed a comprehensive radiobiological response evaluation for the comparison of treatment plans and whereby it validated the method of selection of competing plans for treatment. Also, this work is completed on three particular sites, with two treatment techniques, and by using larger set of data (120 plans).

\section{Conclusion}

This retrospective dosimetric study of IMRT and RA plans, performed on different groups of patients, validated the use of radiobiological method for treatment plan comparison. The comprehensive radiobiological response study endorses the routine plan evaluation by analysis of isodose distribution and DVHs. For the examined patient cohort, a better target coverage is noticed for final plans in each group, where as no statistically significant differences in physical and biological dose indices of OARs were observed. The estimated biological outcome and DVH analysis indicated that the iterative optimization produces least differences between plans for IMRT when compared to RA. The tumor cure or normal tissue complication probabilities were found to be well correlated with the corresponding physical dose indices.

\section{References}

[1] Yu CX. Intensity modulated arc therapy: A new method for delivering conformal radiation therapy. The theory \& practice of intensity modulated radiation therapy. Madison, WI: Advanced Medical Publishing; 1997:107-20.

[2] Mavroidis P, Lind BK, Brahme A. Biologically effective uniform dose (D) for specification, report and comparison of dose response relations and treatment plans. Phys Med Biol. 2001;46(10):2607-2630.

[3] Mohan R, Wang X, Jackson A, et al. The potential and limitations of the inverse radiotherapy technique. Radiother Oncol. 1994;32(3):232-348.

[4] Vineberg KA, Eisbruch A, Coselmon MM, et al. Is uniform target dose possible in IMRT plans in the head and neck? Int J Radiat Oncol Biol Phys. 2002;52(5):1159-1172.

[5] Miften MM, Das SK, Su M, et al. A dose-volume based tool for evaluating and ranking IMRT treatment plans. J Appl Clin Med Phys. 2004;5(4):1-14.

[6] Mayo C, Yorke E, Merchant TE. Radiation associated brainstem injury. Int J Radiat Oncol Biol Phys. 2010;76(3Suppl):S36-S41.

[7] Mayo C, Martel MK, Marks LB, et al. Radiation dose-volume effects of optic nerves and chiasm. Int J Radiat Oncol Biol Phys. 2010;76(3Suppl):S28-S35.

[8] Kirkpatrick JP, van der Kogel AJ, Schultheiss TE. Radiation dose-volume effects in the spinal cord. Int J Radiat Oncol Biol Phys. 2010;76(3Suppl):S42-S49.

[9] Bhandare N, Jackson A, Eisbruch A, et al. Radiation therapy and hearing loss. Int J Radiat Oncol Biol Phys. 2010;76(3Suppl):S50S57.

[10] Emami B, Lyman J, Brown A, et al. Tolerance of normal tissue to therapeutic irradiation. Int J Radiat Oncol Biol Phys. 1991;21(1):109-122.

[11] Rubin P. Law and order of radiation sensitivity: absolute versus relative. In: Vaeth JM, Meyer JL, eds. Frontiers of radiation therapy and oncology. Basel: Karger;1989:7-40.

[12] Deasy JO, Moiseenko V, Marks L, et al. Radiotherapy dose-volume effects on salivary gland function. Int J Radiat Oncol Biol Phys. 2010;76(3Suppl):S58-S63.

[13] Lawton CA, Michalski J, El-Naqa I, et al. RTOG GU Radiation oncology specialists reach consensus on pelvic lymph node volumes for high-risk prostate cancer. Int J Radiat Oncol Biol Phys. 2009;74(2):383-387.

[14] Viswanathan AN, Yorke ED, Marks LB, et al. Radiation dose-volume effects of the urinary bladder. Int J Radiat Oncol Biol Phys. 2010;76(3Suppl):S116-S122.

[15] Michalski JM, Gay H, Jackson A, et al. Radiation dose-volume effects in radiation-induced rectal injury. Int J Radiat Oncol Biol Phys. 2010;76(3Suppl):S123-S129.

[16] Niemierko A. Reporting and analyzing dose distributions: a concept of equivalent uniform dose. Med Phys. 1997;24(1):103-110. 
[17] Thames HD, Zhang M, Tucker SL, et al. Cluster models of dose-volume effects. Int J Radiat Oncol Biol Phys.. 2004;59(5):14911504.

[18] Lyman JT. Complication probability as assessed from dose-volume histograms. Radiat Res Suppl. 1985;8:S13-S19.

[19] Niemierko A, Goitein M. Modeling of normal tissue response to radiation: the critical volume model. Int J Radiat Oncol Biol Phys. 1993;25(1):135-145.

[20] Anbumani S, Raj NA, Prabhakar GS, et al. Quantification of uncertainties in conventional plan evaluation methods in Intensity Modulated Radiation Therapy. J BUON. 2014;19(1):297-303.

[21] Gay HA, Niemierko A. A free program for calculating EUD-based NTCP and TCP in external beam radiotherapy. Physica Medica. 2007;23(3):115-125.

[22] Niemierko A. A generalized concept of equivalent uniform dose (EUD). Med Phys. 1999;26(6):1100.

[23] Oinam AS, Singh L, Shukla A, et al. Dose volume histogram analysis and comparison of different radiobiological models using inhouse developed software. J Med Phys. 2011;36(4):220-229.

[24] Rana S, Cheng C. Radiobiological impact of planning techniques for prostate cancer in terms of tumor control probability and normal tissue complication probability. Ann Med Health Sci Res. 2015;4(2):167-172.

[25] Jaganathan A, Tiwari M, Phansekar R, et al. Intensity-modulated radiation to spare neural stem cells in brain tumors: a computational platform for evaluation of physical and biological dose metrics. J Cancer Res Ther. 2011;7(1):58-63.

[26] Kehwar TS. Analytical approach to estimate normal tissue complication probability using best fit of normal tissue tolerance doses into the NTCP equation of the linear quadratic model. J Cancer Res Ther. 2005;1(3):168-179.

[27] Rana S, Rogers K. Radiobiological evaluation of dose calculation algorithms in Rapid Arc planning of esophageal cancer treatment plans. J Solid Tumors. 2013;3(3):44-52.

[28] Akpati HC, Kim C, Kim B, et al. Unified dosimetry index (UDI): a figure of merit for ranking treatment plans. J Appl Clin Med Phys. 2008;9(3):99-108. 